\title{
Comparison of Efficacy of Clotrimazole 1\% Cream with Sertaconazole 2\% Cream in Treatment of Adult Seborrheic Dermatitis
}

\author{
Pedram Alirezaei ${ }^{1 *}$ (iD , Nasrin Jiryaee ${ }^{2}$, Ali Abbas Shabbak ${ }^{3}$ \\ ${ }^{I}$ Assistant Professor, Psoriasis Research Center, Hamadan University of Medical Sciences, Hamadan, Iran \\ 2 Assistant Professor, Department of Social Medicine, School of Medicine, Hamadan University of Medical Sciences, \\ Hamadan, Iran \\ ${ }^{3}$ Resident, Department of Dermatology, School of Medicine, Hamadan University of Medical Sciences, Hamadan, Iran
}

* Corresponding Author: Pedram Alirezaei, Psoriasis Research Center, Hamadan University of Medical Sciences, Hamadan, Iran.Email:Prc@umsha.ac.ir

\begin{tabular}{|c|c|}
\hline \multirow[b]{2}{*}{$\begin{array}{l}\text { Received: } 16.05 .2019 \\
\text { Accepted: } 19.08 .2019\end{array}$} & Abstract \\
\hline & \multirow[b]{2}{*}{$\begin{array}{l}\text { Background and Objective: Seborrheic dermatitis is a common } \\
\text { inflammatory skin disease characterized by erythema, scaling, and pruritus. } \\
\text { Antifungals are among the most commonly prescribed drugs for the } \\
\text { treatment of this disease. In this regard, the present study investigated the } \\
\text { efficacy of two medications including clotrimazole } 1 \% \text { and sertaconazole } \\
2 \% \text { in the treatment of adult seborrheic dermatitis. } \\
\text { Materials and Methods: This double-blinded randomized clinical trial was } \\
\text { performed on } 100 \text { patients over the age of } 18 \text { with seborrheic dermatits } \\
\text { referring to Farshchian hospital, Hamadan, Iran. Patients were randomly } \\
\text { assigned to two groups. The participants in one group received sertaconazole } \\
\text { twice a day for four weeks and another group received clotrimazole within } \\
\text { this period of time. After two weeks, the patients were examined in terms of } \\
\text { clinical symptoms and side effects of medications. Moreover, they were } \\
\text { reexamined for recurrence of disease after the completion of treatment and } \\
\text { four weeks later. Furthermore, the level of patients' satisfaction was } \\
\text { evaluated four weeks after treatment. } \\
\text { Results: Mean and standard deviation of Scoring Index } 7.16 \pm 2.20 \text { and } \\
6.45 \pm 2.10 \text { before treatment }(\mathrm{P}=0.184), 4.08 \pm 1.82 \text { and } 2.78 \pm 2.14 \text { two } \\
\text { weeks after treatment }(\mathrm{P}<0.001 \text { ), and } 2.62 \pm 2.13 \text { and } 0.89 \pm 1.09 \text { four weeks } \\
\text { after the end of treatment }(\mathrm{P}<0.001 \text { ). Additionally, the relapse rate in } \\
\text { clotrimazole and sertaconazole groups was found to be } 32 \% \text { and } 21.7 \% \text {, } \\
\text { respectively. Furthermore, the complication rate was reported as } 8 \% \text { and } \\
8.5 \% \text { in clotrimazole group and sertaconazole group, respectively (P=0.220). } \\
\text { Moreover, the rate of good satisfaction in clotrimazole and sertaconazole } \\
\text { groups was reported as } 38 \% \text { and } 71.1 \% \text { ( }<0.001 \text { ), respectively. } \\
\text { Conclusion: Based on the results of the study, the frequency of } \\
\text { complications and recurrence of disease in patients treated with } \\
\text { sertaconazole was comparable to treatment with clotrimazole. However, } \\
\text { sertoconazole was found to be more effective and bring more satisfaction to } \\
\text { patients. }\end{array}$} \\
\hline $\begin{array}{l}\text { How to Cite this Article: } \\
\text { Alirezaei P, Jiryaee N, Shabbak } \\
\text { AA. Comparison of Efficacy of } \\
\text { Clotrimazole } 1 \% \text { Cream with } \\
\text { Sertaconazole } 2 \% \text { Cream in } \\
\text { Treatment of Adult Seborrheic } \\
\text { Dermatitis. Avicenna J Clin } \\
\text { Med. 2019; 26(2): } 67-74 \text {. DOI: } \\
\text { 10.21859/ajcm.26.2.67 }\end{array}$ & \\
\hline & \\
\hline
\end{tabular}


dof: $10.21859 /$ ajcm.26.2.67

مقاله يزوهشى

\title{
مقايسه اثربخشى كرم كلوتريمازول ا درصد با كرم سرتاكونازول Y درصد در درمان درماتيت سبورئيك بزركسالان
}

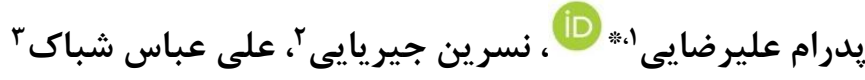

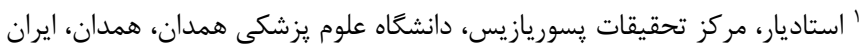

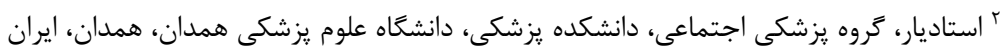

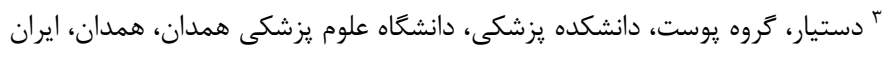

* نويسنده مسئول: يدرام عليرضايى، مركز تحقيقات يسوريازيس، دانشكاه علوم يزشكى همدان، همدان، ايران. ايميل: Prc umsha.ac.ir

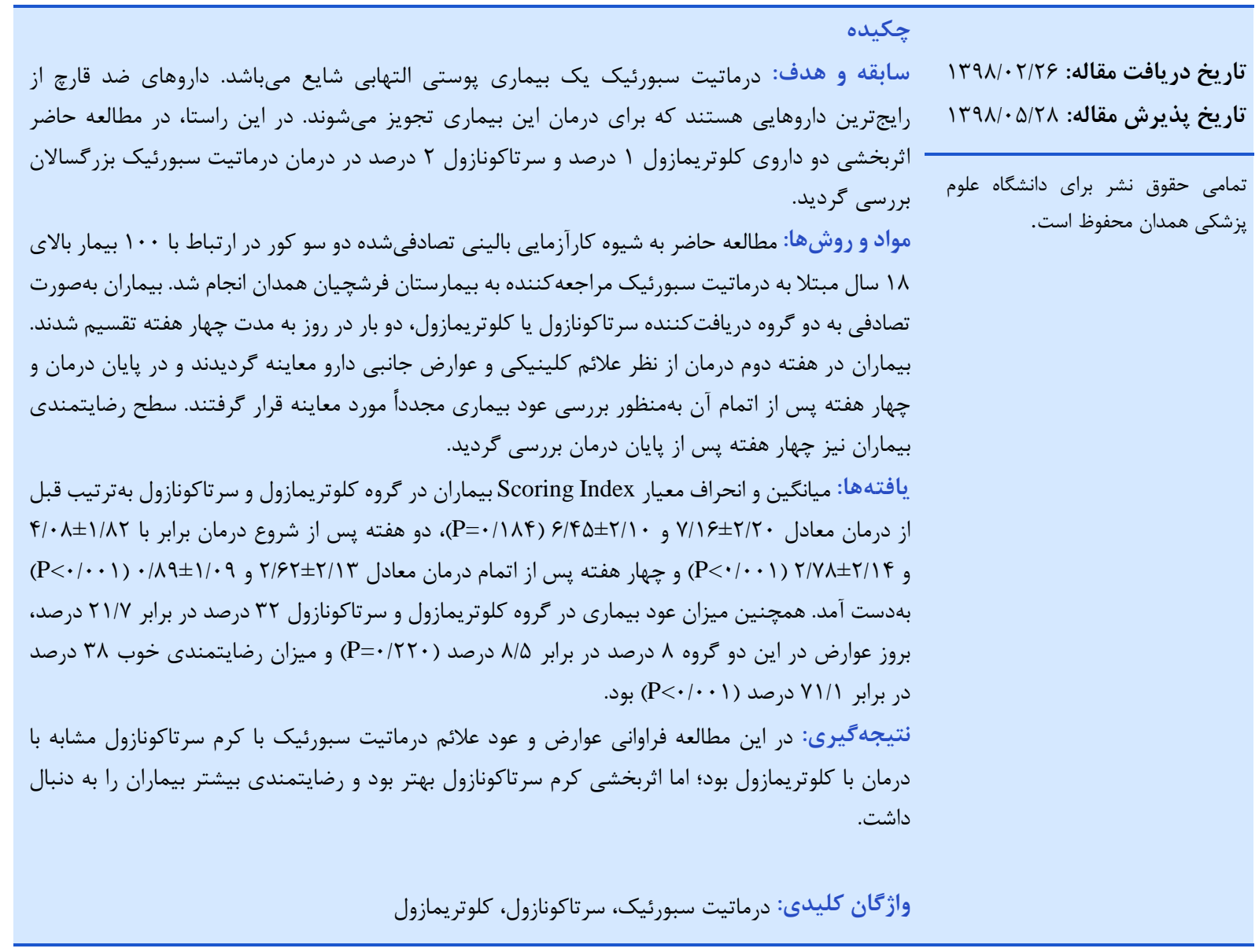

مقلdمه

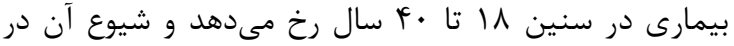

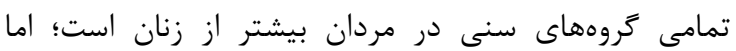

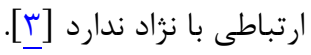

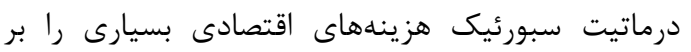

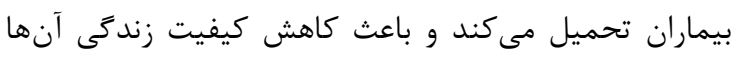

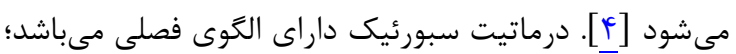

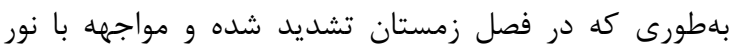

درماتيت سبورئيك (SD: Seborrheic Dermatitis) يك (يك

بيمارى شايع التهابى است كه غدد سبابه را دركير مى كند [1 إنيك.

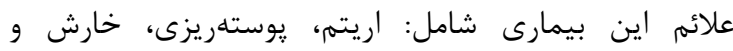

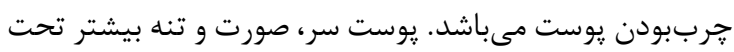

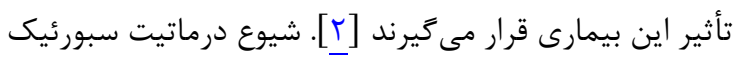

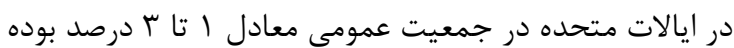

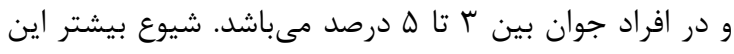




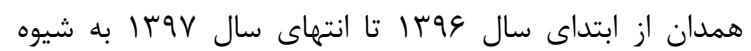

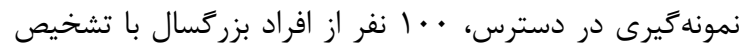

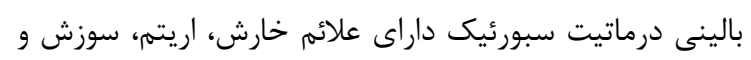

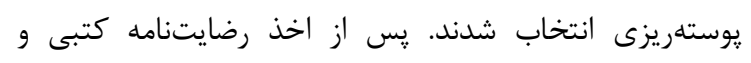

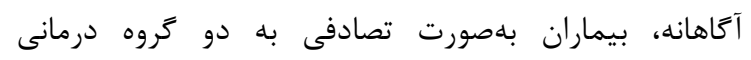

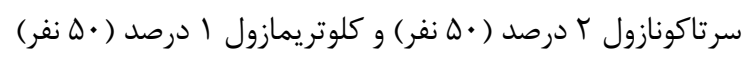
تقسيم گرديدند.

بلهنظور عدم اطلاع تجويزكننده و بيمار از نوع دارو، كرمها

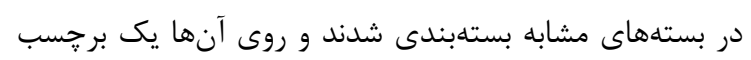

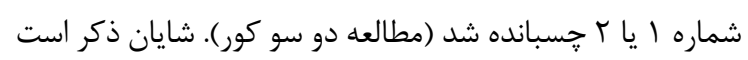
كه محتواى بستهها از نظر رنخ، بو و قوام كاملاً مشابه بودند.

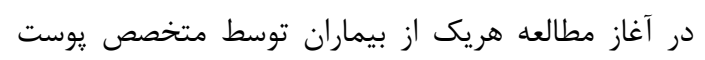

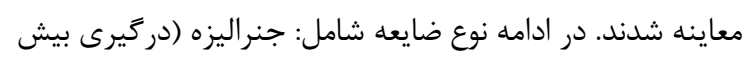

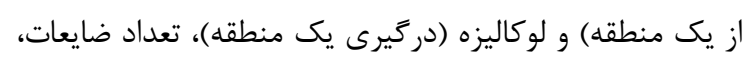

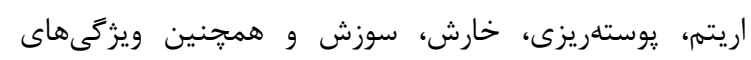

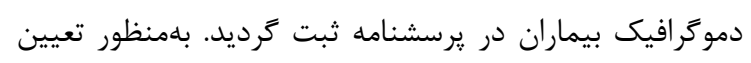

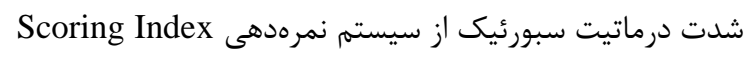

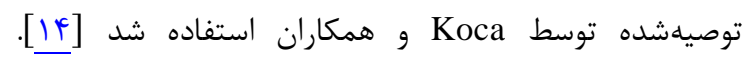

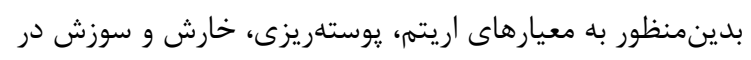

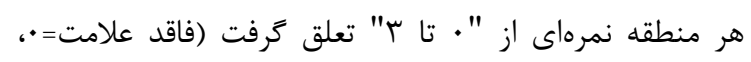

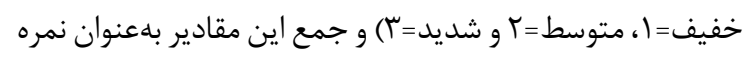
بيمار ثبت گرديد.

شيوه درمان در هر دو تروه بلهورت موضعى دئ، دو بار در روز

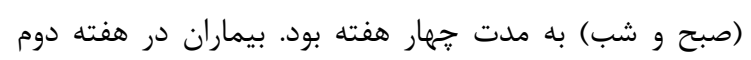

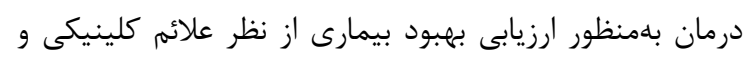

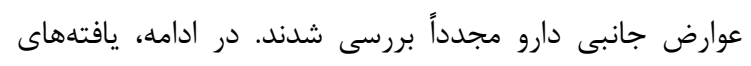

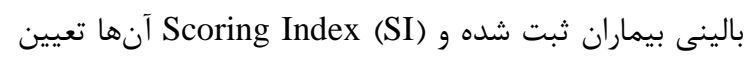
مى

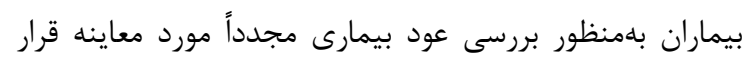

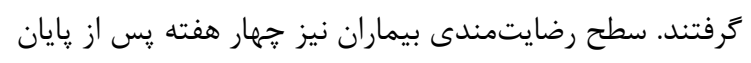

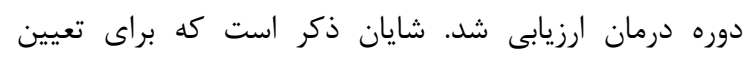

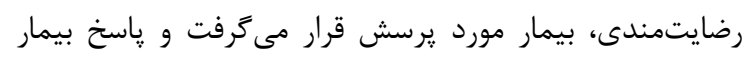

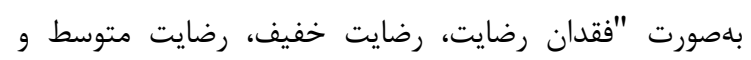
رضايت خوب" طبقهبندى مىشد. معيارهاى ورود به مطالعه عبارت بودند از: ابتلا به درماتيت

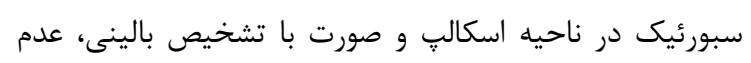

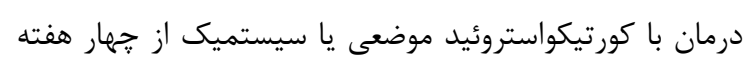

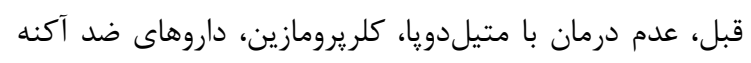

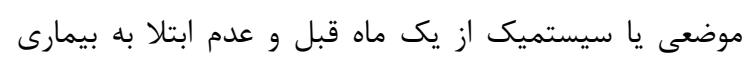

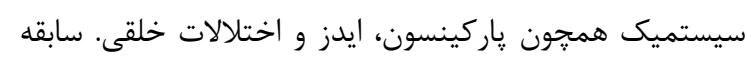

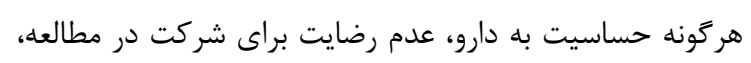

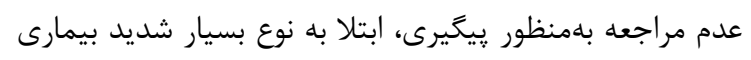

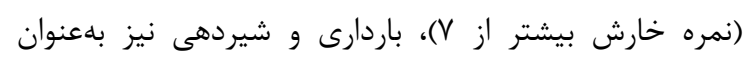

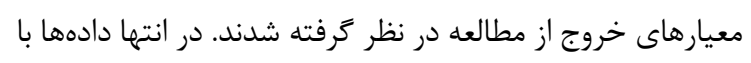

آفتاب در تابستان باعث بهبود نسبى علائم بيمارى مى

فعاليت غدد سبابه با توجه به محل آناتوميك، جنس و سن متفاوت است. غدد سباسه هنگام تولد وجود دارند و ترشح سبوم

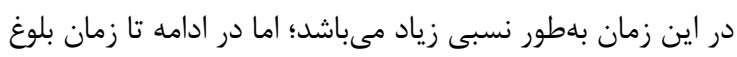

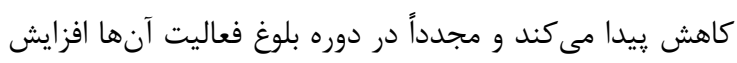

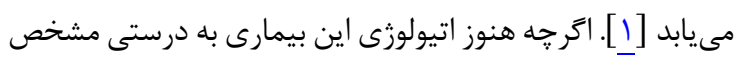

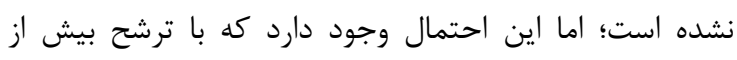

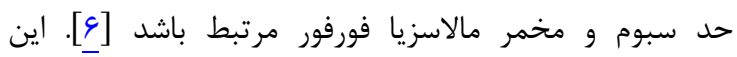

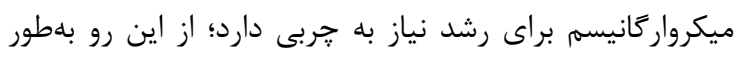

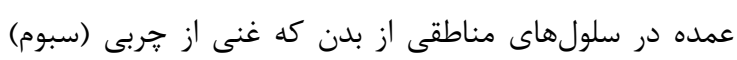

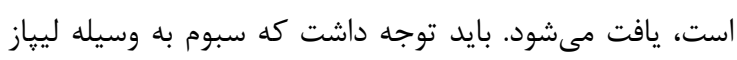

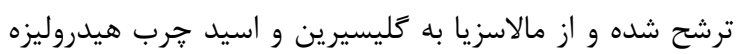

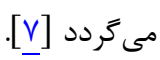

دو گروه دارويى مهام درد درمان درمان دماتيـت سبورئيك،

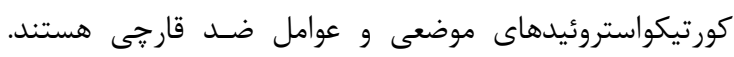

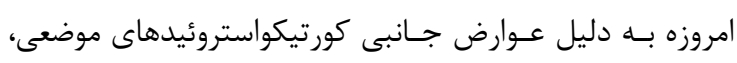

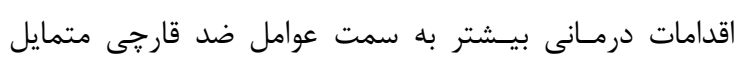

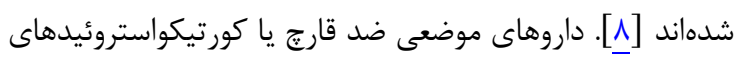

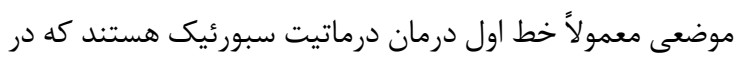

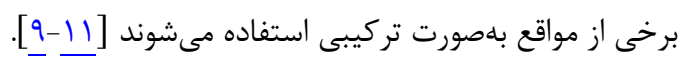

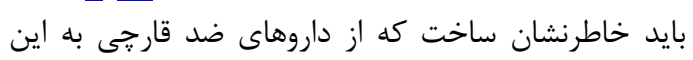

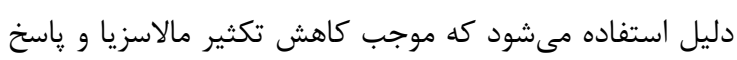

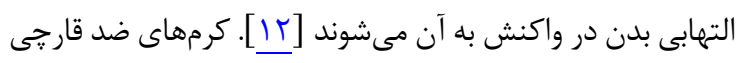

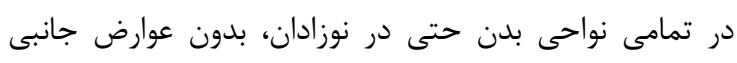

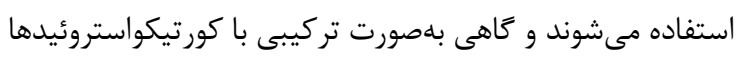

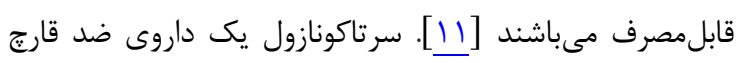

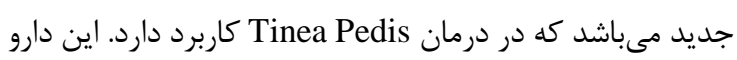

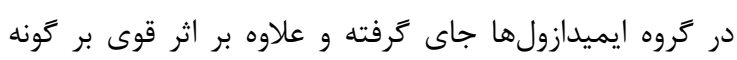

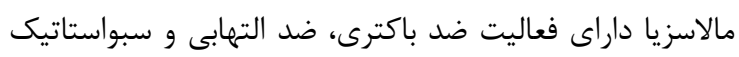

مىباشد [11ا].] با توجه به شيوع بالاى درماتيت سبورئيك و بار اقتصادى و

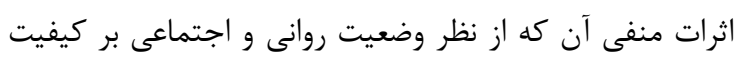

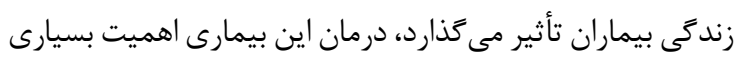

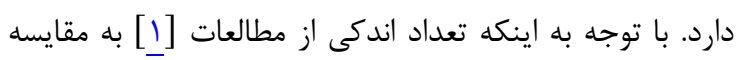

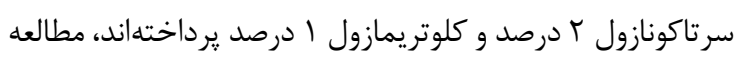

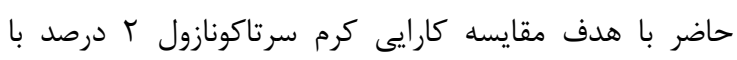

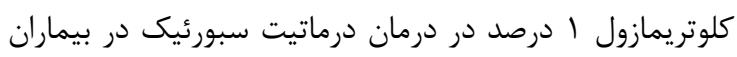
بالاى 1^ا سال انجام شد.

\section{مواد و روشها}

مطالعه حاضر بلصورت كار آزمايى بالينى تصادفىشده دو سو

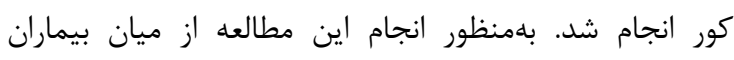

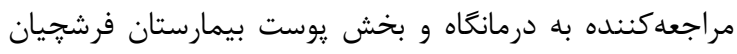


بهطور معنادارى كمتر از گروه درمانى كلوتريمازول بود

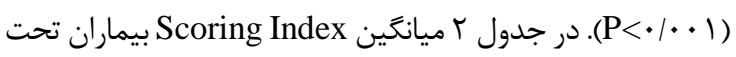
درمان با كرم كلوتريمازول و سرتاكونازول در آغاز درمان، دو هفته

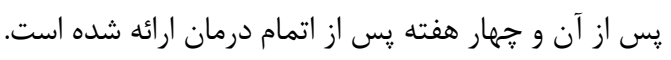

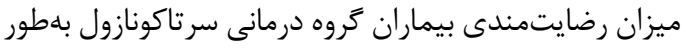

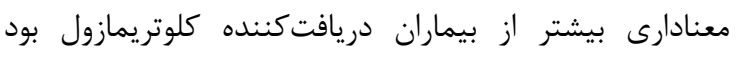

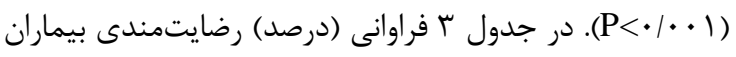

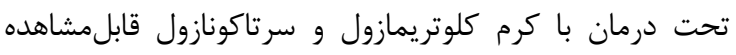
مىباشد.

فراوانى عود بيمارى طى جهار هفته يس از شروع درمان

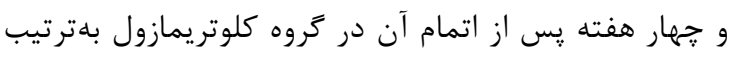

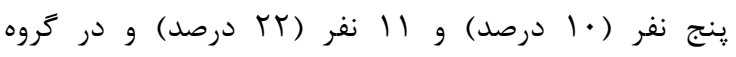

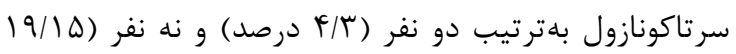

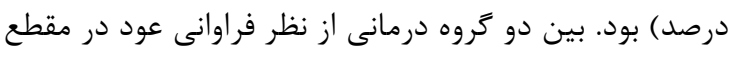

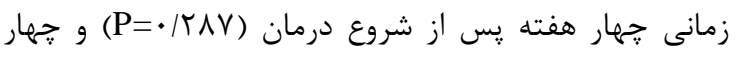

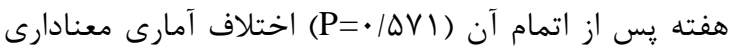
مشاهده نشد.

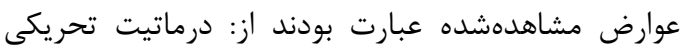

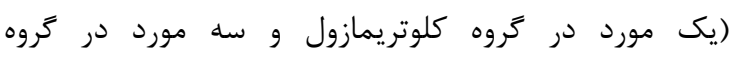

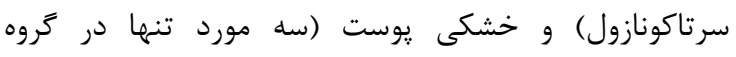

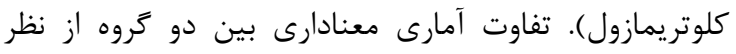

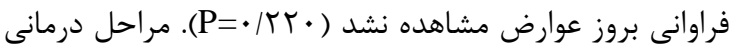

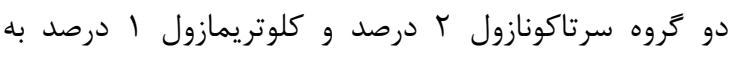
تفكيك در فلوجارت ا نشان داده شده است
استفاده از نرمافزار SPS 16 در سطح اطمينان ه9 درصد تجزيه

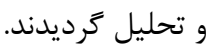

\section{يافته ها}

در اين مطالعه در مجموع · ․ بيمار مورد بررسى قرار

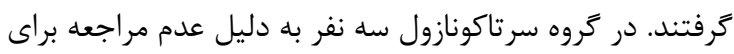

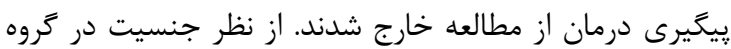

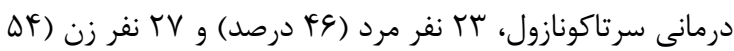

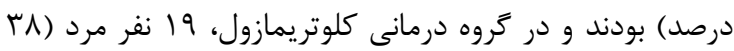

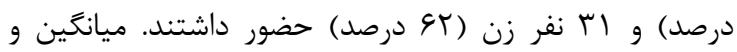

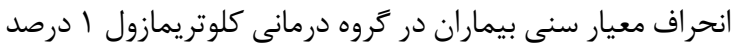

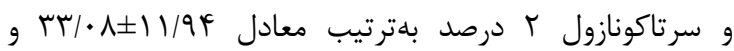

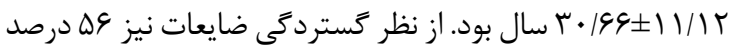

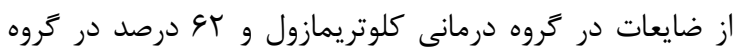

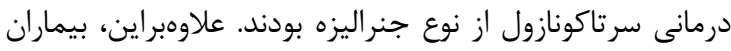

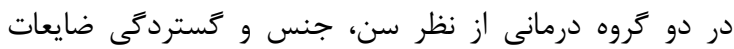

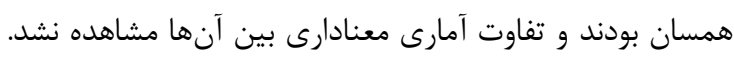

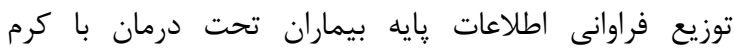

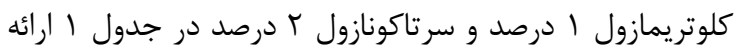
شده است. بيماران در دو كروه درمانى كلوتريمازول و سرتاكونازول از نظر

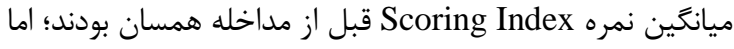

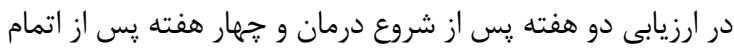

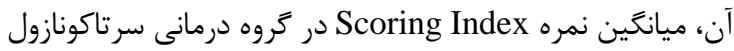

جدول ا: توزيع فراوانى اطلاعات يايه بيماران تحت درمان با كرم كلوتريمازول ا درصد و سرتاكونازول ب درصد

\begin{tabular}{|c|c|c|c|}
\hline \multirow[b]{2}{*}{ سطح معنادارى } & \multicolumn{2}{|c|}{ كروه درمانى } & \multirow{2}{*}{ منسير (سطوح متغير ) } \\
\hline & سر تاكونازول Y درصد (درصد) & كلوتريمازول ادرصد (درصد & \\
\hline \multirow{2}{*}{$\cdot|f| V^{*}$} & (F\&) Tr & $($ (ז) 19 & مرد \\
\hline & $(\Delta F) Y V$ & I I & زن \\
\hline$\cdot / r 99^{* *}$ & $(11 / 1 T) r \cdot 199$ & (1)/qF) $r / \cdot \wedge$ & ميانگين سنى (انحراف معيار ) \\
\hline \multirow{2}{*}{$\cdot|\Delta F|^{*}$} & $($ (ז) 19 & (FY) Tr & كستر دكى ضايعات \\
\hline & (GT) rI & 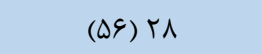 & جنراليزه \\
\hline
\end{tabular}

T-Student آزمون مجذور كاى *قاه

جدول r: مقايسه ميانغين (انحراف معيار) Scoring Index بيماران تحت درمان با كرم كلوتريمازول ا درصد و سرتاكونازول r درصد در آغاز درمان،

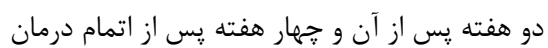

\begin{tabular}{|c|c|c|c|}
\hline \multirow[b]{2}{*}{ سطح معنادارى } & كانى & ترو & \multirow[b]{2}{*}{ زمان ارزيابى } \\
\hline & ميانًين (انحر اف معازول ب درصد ) & ميانتَين (انحراف معيار ) ملرول & \\
\hline$\cdot /\left.\Lambda \Lambda\right|^{*}$ & $(T / 1 \cdot) \varepsilon / F \Delta$ & $(Y / T \cdot) V / 1 \varphi$ & شروع درمان \\
\hline$<\cdot|\cdot \cdot|^{\text {絭常 }}$ & $(Y / I F) T / V \Lambda$ & $(1 / \Lambda r) Y / \cdot \Lambda$ & دو هفته يس از آغاز درمان \\
\hline$<\left.\cdot 1 \cdot \cdot\right|^{\text {***\% }}$ & $(1 / \cdot 9) \cdot 119$ & $(Y / I T) Y / g T$ & جهار هفته يس از اتمام درمان \\
\hline
\end{tabular}

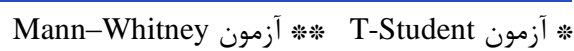


جدول ب: مقايسه فراوانى (درصد) رضايتمندى بيماران تحت درمان با كرم كلوتريمازول و سرتاكونازول

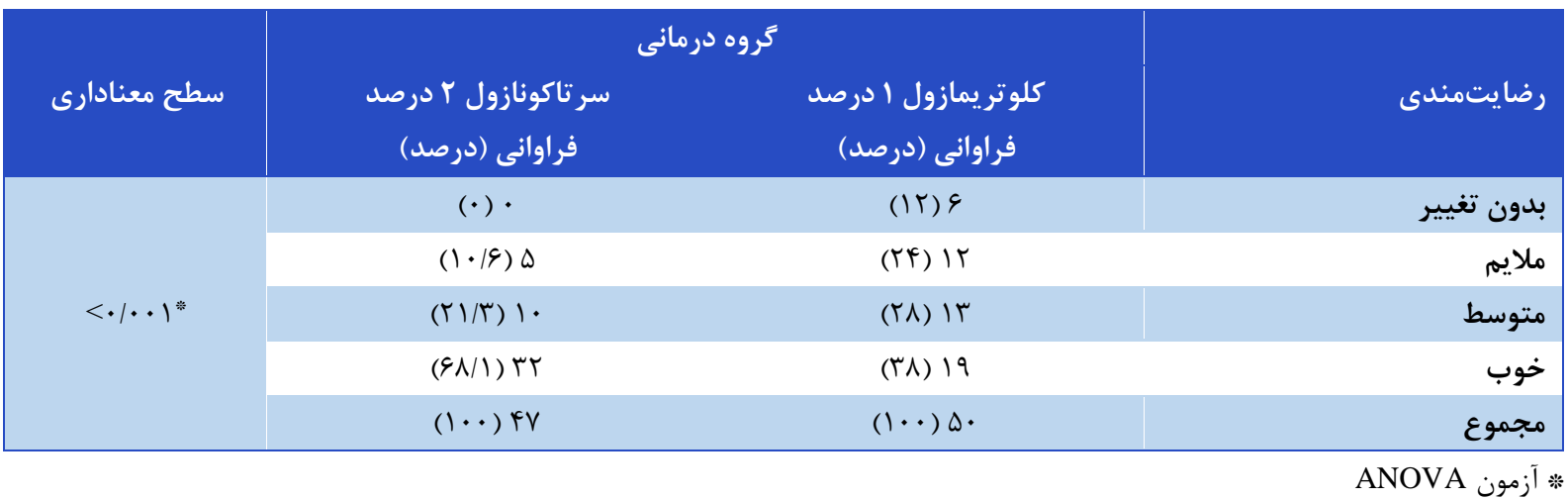

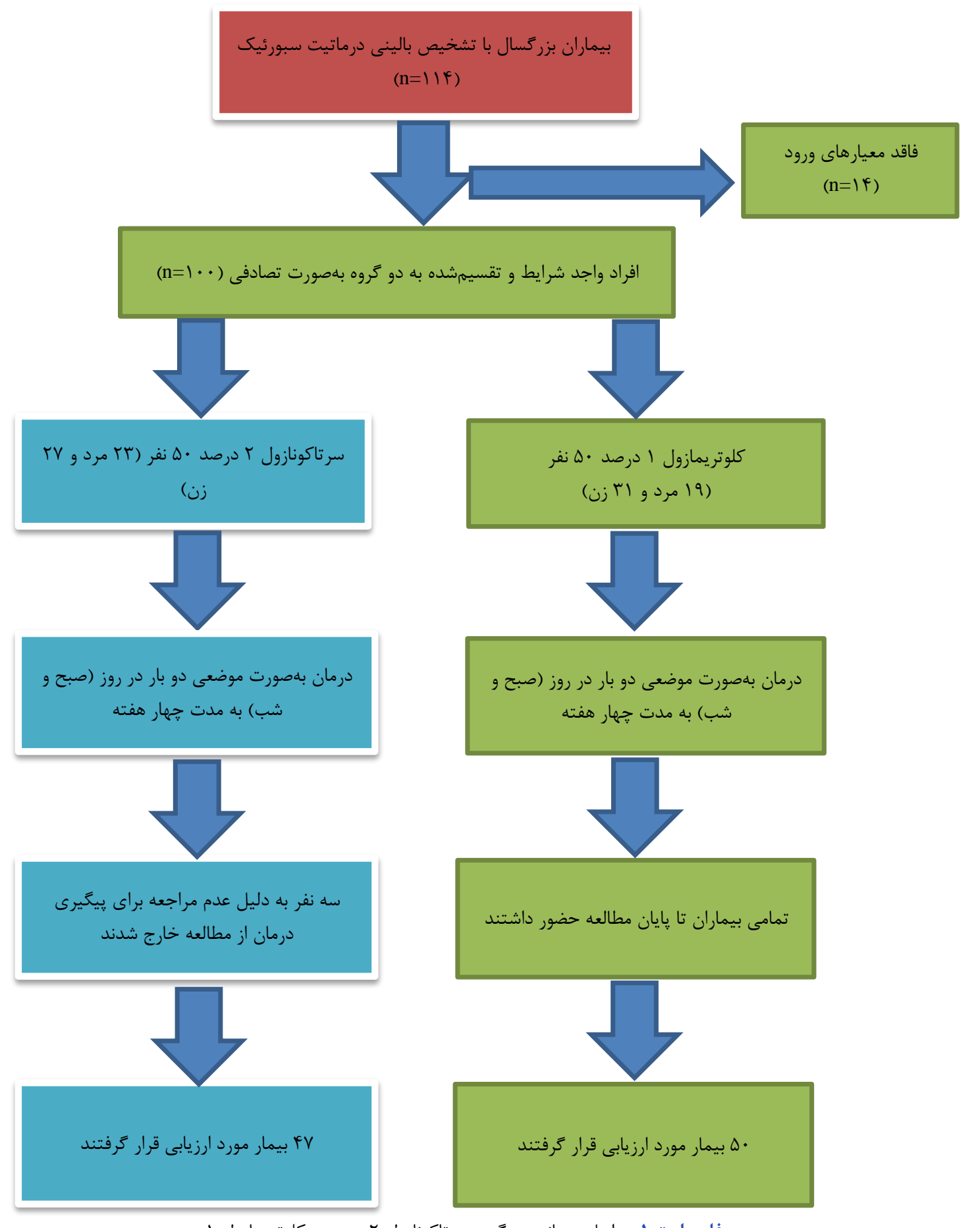

فلو جارت ا: مراحل درمانى دو كروه سرتاكونازول r درصد و كلوتريمازول ا درصد 
اين مطالعه مىتوان كفت كه اكرجه روغن شترمرغ در درمان

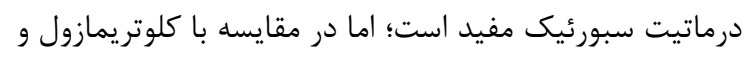

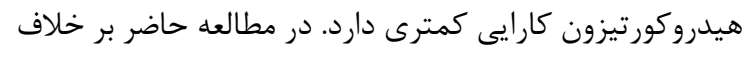

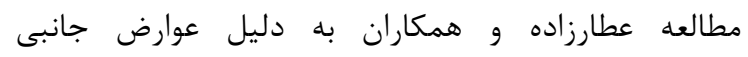

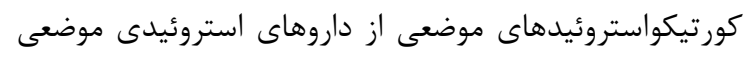

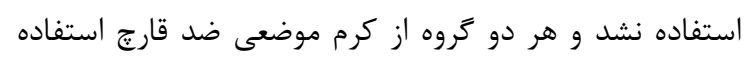

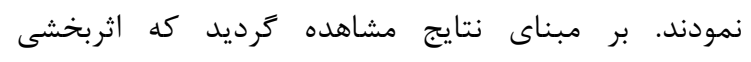

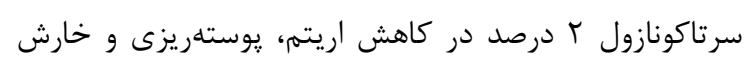

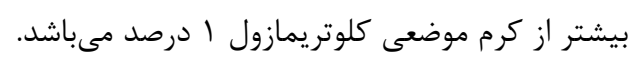

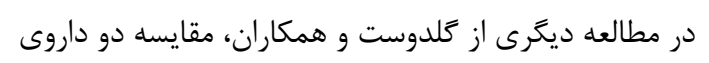

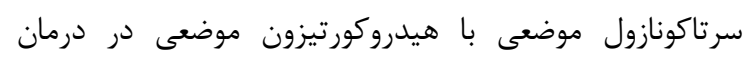

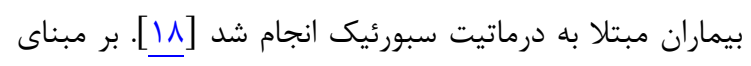

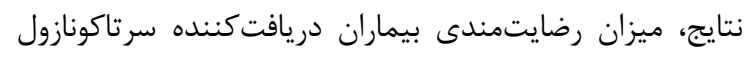

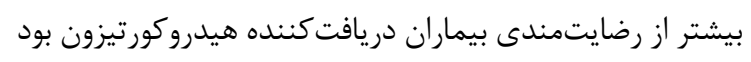

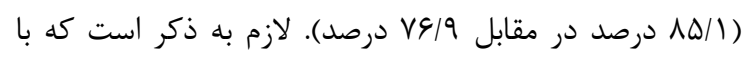

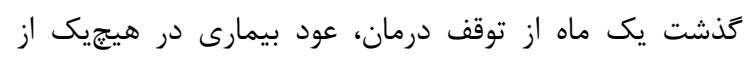

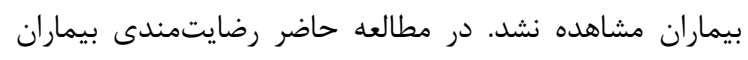

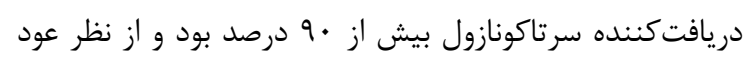

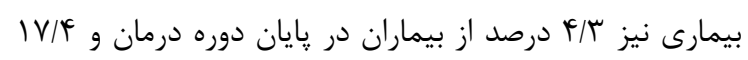

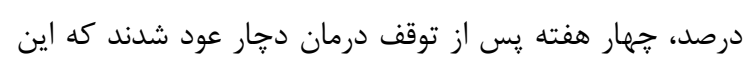

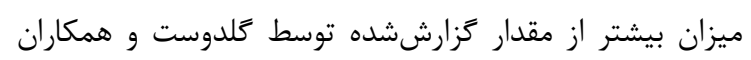

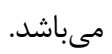

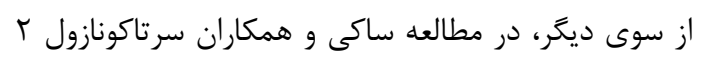

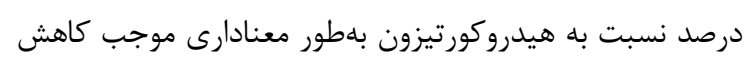

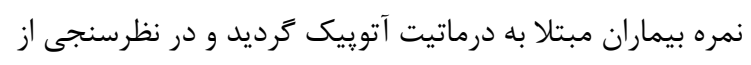

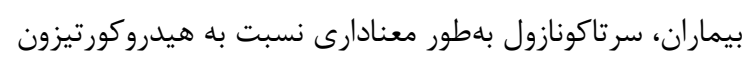

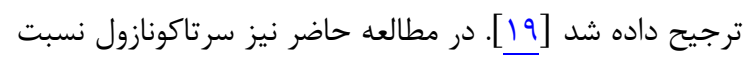

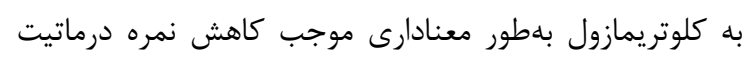

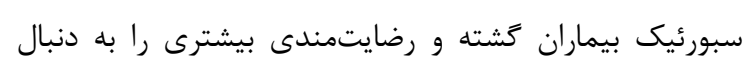

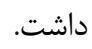

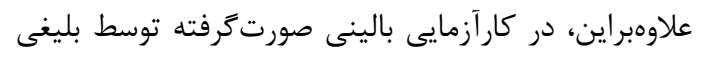

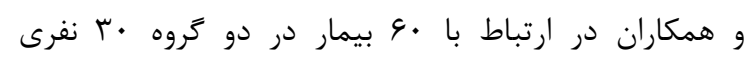

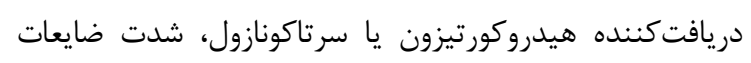

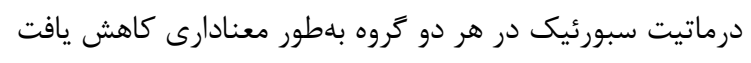

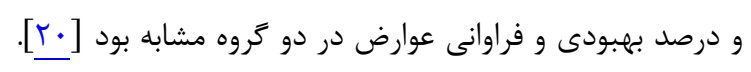

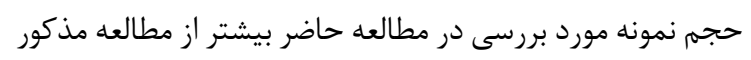

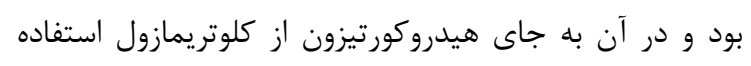

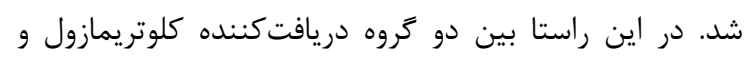

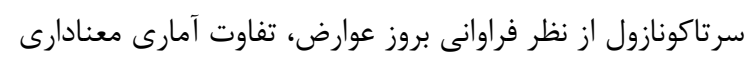

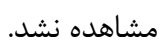

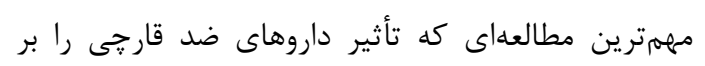

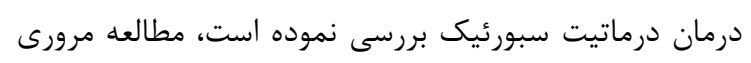

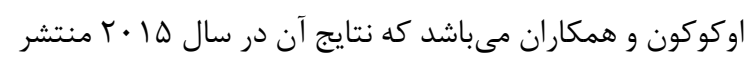

كلدوست و همكاران در مطالعه خود كارايى يماد سر تاكونازول

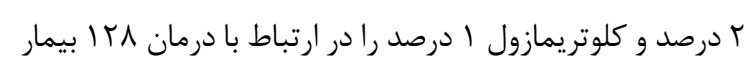

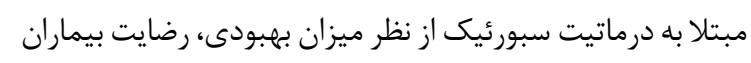

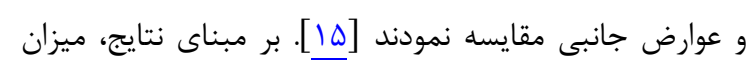

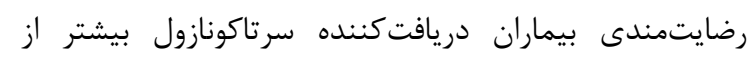

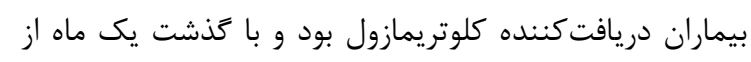

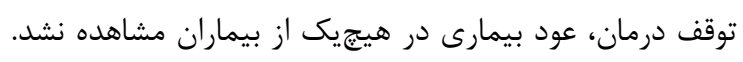

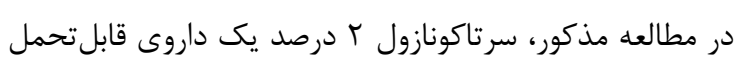

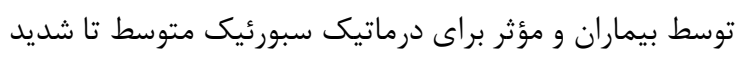

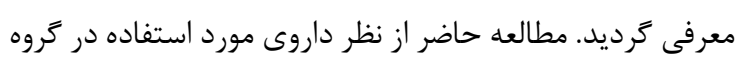

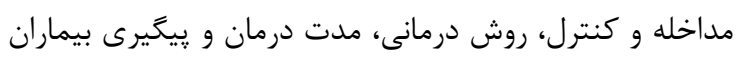

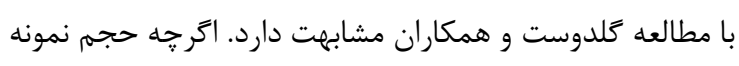

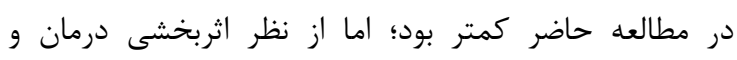

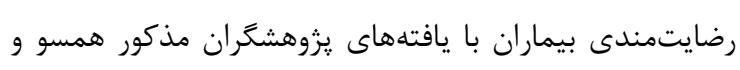
همجرت مىباشد.

همجنين در مطالعه صورت كرفته توسط لوتى ونى و همكاران

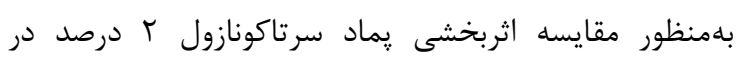

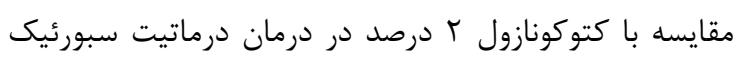

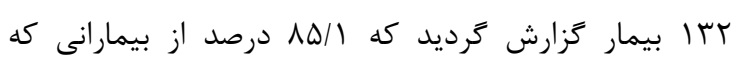

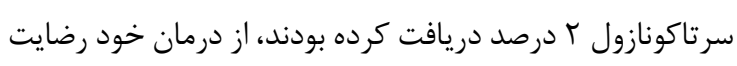

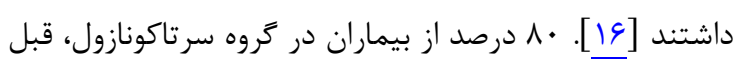

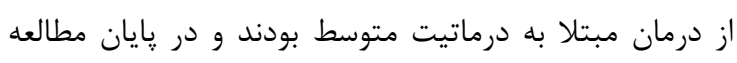

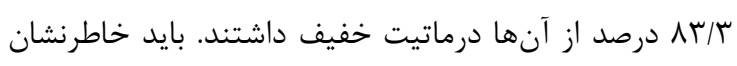

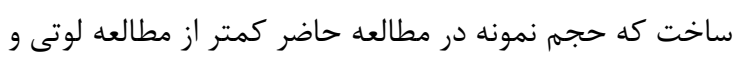

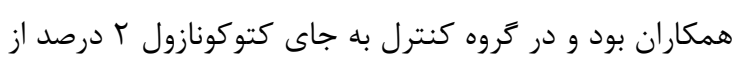

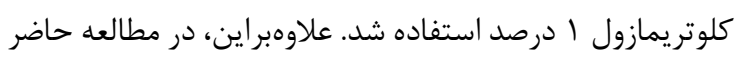

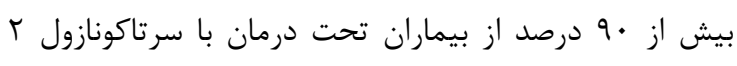

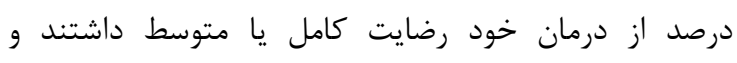

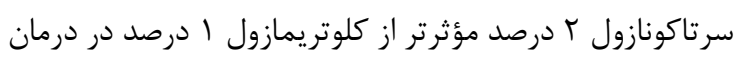

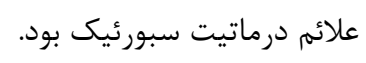
در كارآزمايى بالينى انجامشده توسط عطارزاده و همكاردان

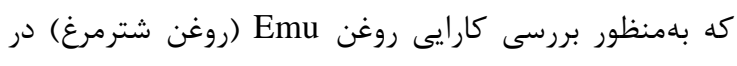

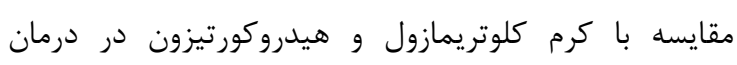

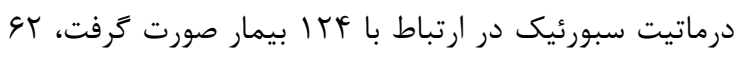

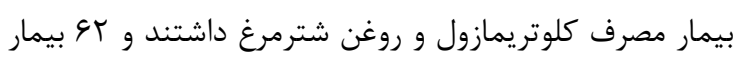

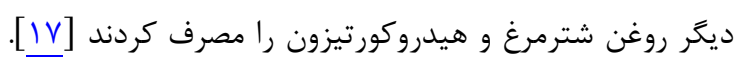

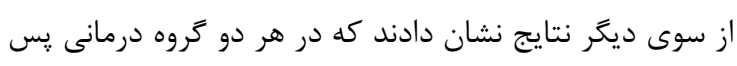

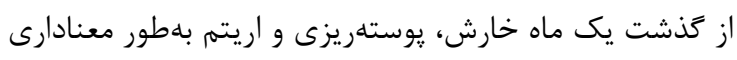

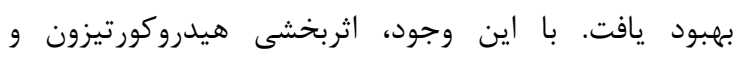

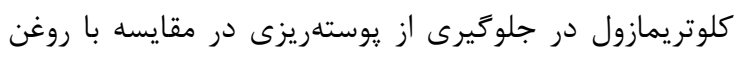

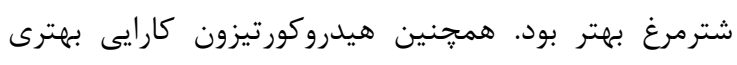

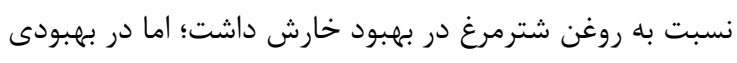

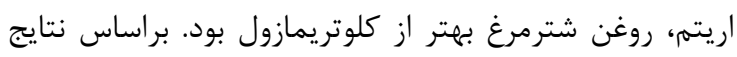


بود. ميزان رضايتمندى بيماران تحت درمان با كرم سرتاكونازول

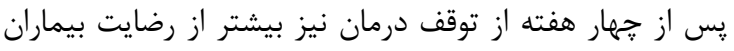
تحت درمان با كرم كلوتريمازول بود. با توجه به نقش مخمر مالاسـزيا در ايجاد درماتيت سبورئيك دورد

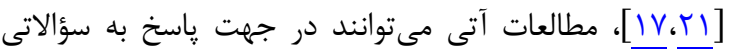

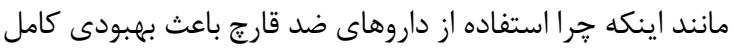

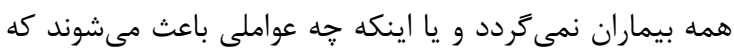

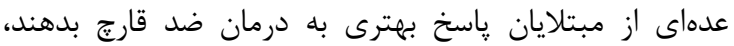
طراحى گردد.

\section{تشكر و قدر فاذى}

مقاله حاضر برگرفته از ياياننامه دوره دكترى تخصصى

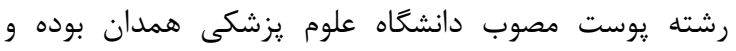

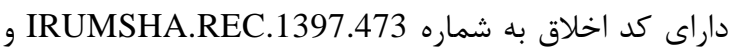
IRCT201701299014N141 محترم تحقيقات و فناورى دانشعاه علوم يزشكى همدان به دليل همكارى در راستاى تصويب اين طرح يزوهشى و نيز از كاركنان

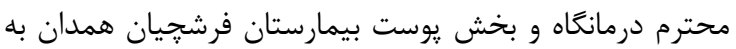

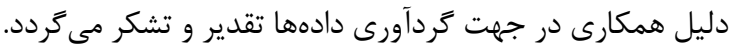

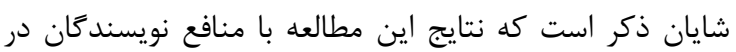

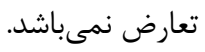

\section{REFERENCES}

1. Bolognia JL, Jorizzo JL, Rapini RP. Dermatology. $3^{\text {rd }}$ ed. Philadelphia: Elsevier; 2012.

2. Gary G. Optimizing treatment approaches in seborrheic dermatitis. J Clin Aesthet Dermatol. 2013;6(2):44-9. PMID: 23441240

3. Holden CA, Berth-Jones J. Eczema, lichenification, prurigo and erythroderma. Rook's textbook of dermatology. $7^{\text {th }}$ ed. New York: Blackwell Science; 2004.

4. Szepietowski JC, Reich A, Wesolowska-Szepietowska E, Baran E. Quality of life in patients suffering from seborrheic dermatitis: influence of age, gender and education level. Mycoses. 2009;52(4):357-63. PMID: 18793259 DOI: 10.1111/j.1439-0507.2008.01624.x

5. Tegner E. Seborrhoeic dermatitis of the face induced by PUVA treatment. Acta Derm Venereol. 1983;63(4):335-9. PMID: 6195866

6. Jean LB, Joseph LJ, Rapini RP, Thomas DH, Mascaro JM, Anthony JM. Dermatology. $2^{\text {nd }}$ ed. Spain: Elsevier; 2008.

7. Dawson Jr TL. Malassezia globosa and restricta: breakthrough understanding of the etiology and treatment of dandruff and seborrheic dermatitis through whole-genome analysis. J Investig Dermatol Symp Proc. 2007;12(2):15-9. PMID: 18004291 DOI: 10.1038/sj.jidsymp.5650049

8. Dorn M, Rocehnert K. Dimorphism of Pityrosporum orbicular in a defind culture medium. J Invest Dermatol. 1977;62(2):244-8. PMID: 881572 DOI: 10.1111/15231747.ep12506384

9. Bikowski J. Facial seborrheic dermatitis: a report on current status and therapeutic horizons. J Drugs Dermatol. 2009; 8(2):125-33. PMID: 19213227

10. Naldi L, Rebora A. Clinical practice. Seborrheic dermatitis. $N$ Engl J Med. 2009;360(4):387-96. PMID: 19164189 DOI: 10.1056/NEJMcp0806464

11. Ortonne JP, Nikkels AF, Reich K, Ponce Olivera RM, Lee $\mathrm{JH}$, Kerrouche N, et al. Efficacious and safe management of moderate to severe scalp seborrhoeic dermatitis using clobetasol propionate shampoo $0.05 \%$ combined with ketoconazole shampoo $2 \%$ : a randomized, controlled study.
گرديد [11 [1]. براساس نتايج اين مطالعه، مؤثرترين داروها در

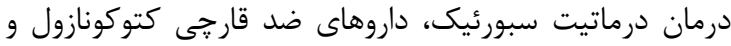

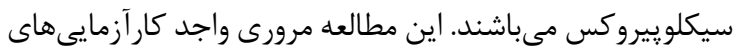

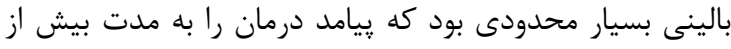

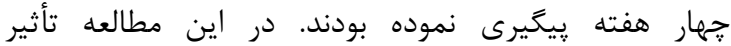

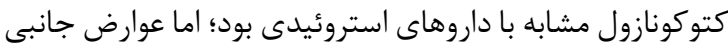

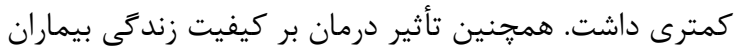

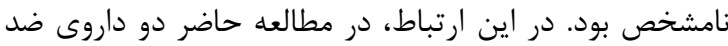

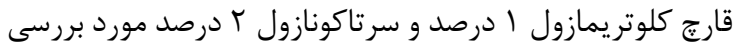

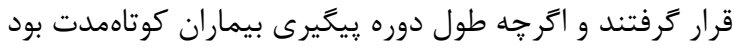

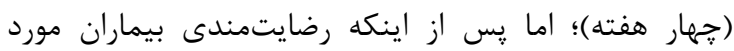

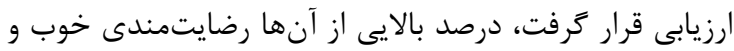

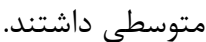

\section{نتيجه تَيرى}

داروهاى ضد قارج موضعى شامل كلوتريمازول و سرتاكونازول

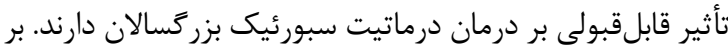

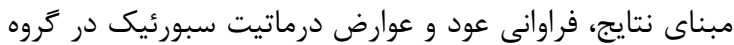

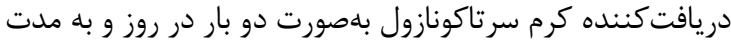

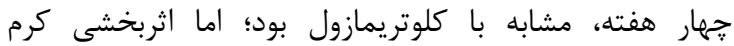
سرتاكونازول در بهبود علائم بيمارى بيشتر از كرم كلوتريمازول

Br J Dermatol. 2011;165(1):171-6. PMID: 21707573 DOI: 10.1111/j.1365-2133.2011.10269.x

12. Nenoff $P$, Haustein UF. In vitro susceptibility testing of Malassezia furfur against rilopirox. Skin Pharmacol. 1997;10(5-6):275-80. PMID: 9449166

13. Okokon EO, Verbeek JH, Ruotsalainen JH, Ojo OA, Bakhoya VN. Topical antifungals for seborrhoeic dermatitis. Cochrane Database Syst Rev. 2015;5:CD008138. PMID: 25933684 DOI: 10.1002/14651858.CD008138.pub

14. Koca R, Altinyazar HC, Eştürk E. Is topical metronidazole effective in seborrheic dermatitis? A double-blind study. Int J Dermatol. 2003;42(8):632-5. PMID: 12890109 DOI: 10.1046/j.1365-4362.2003.01981.x

15. Goldust M, Rezaee E, Rouhani S. Double blind study of sertaconazole $2 \%$ cream vs. clotrimazole $1 \%$ cream in treatment of seborrheic dermatitis. Ann Parasitol. 2013; 59(1):25-9. PMID: 23829055

16. Lotti T, Goldust M, Rezaee E. Treatment of seborrheic dermatitis, comparison of sertaconazole $2 \%$ cream versus ketoconazole $2 \%$ cream. J Dermatolog Treat. 2013; 182(4):703-6. PMID: 23441833 DOI: 10.3109/09546634. 2013.777154

17. Attarzadeh Y, Asilian A, Shahmoradi Z, Adibi N. Comparing the efficacy of Emu oil with clotrimazole and hydrocortisone in the treatment of seborrheic dermatitis: a clinical trial. $J$ Res Med Sci. 2013;18(6):477-81. PMID: 24250695

18. Goldust M, Rezaee E, Masoudnia S, Raghifar R. Clinical study of sertaconazole $2 \%$ cream vs. hydrocortisone $1 \%$ cream in the treatment of seborrheic dermatitis. Ann Parasitol. 2013;59(3):119-23. PMID: 24881281

19. Saki N, Jowkar F, Alyaseen S. Comparison of sertaconazole $2 \%$ cream versus hydrocortisone $1 \%$ ointment in the treatment of atopic dermatitis. J Dermatolog Treat. 2013; 24(6):447-9. PMID: 23470235 DOI: $10.3109 / 09546634$. 2013.782384

20. Balighi K, Ghodsi SZ, Daneshpazhooh M, Ghale-Baghi S, Nasimi M, Azizpour A. Hydrocortisone 1\% cream and sertaconazole $2 \%$ cream to treat facial seborrheic 
dermatitis: A double-blind, randomized clinical trial. Int $J$ Womens Dermatol. 2017;3(2):107-10. PMID: 28560305 DOI: $10.1016 /$ j.ijwd.2016.11.008
21. Rippon JW. Medical mycology; the pathogenic fungi and the pathogenic actinomycetes. $3^{\text {rd }}$ ed. Philadelphia: Saunders; 1988. P. 155. 